\title{
Error evaluation of thermistor linearizing circuits
}

\author{
Daniel Slomovitz and José Joskowicz \\ UTE Laboratory, Paraguay 2385, Montevideo, Uruguay
}

Received 25 January 1990, in final form 9 July 1990, Accepted for publication 8 August 1990

Abstract. A simple designing method for linearizing circuits, based on one resistor, is presented. The errors caused by this circuit are calculated, considering the temperature range and the thermistor parameters. These errors are used as a basis for comparing the quality of active linearizing circuits. In many cases, complex circuits recently proposed have similar errors to single resistor linearizing circuits.

It is shown that the exponential approximation is one of the most important error sources. We propose to use more precise models. The relationship between the parameters of the exponential model and those of other models is discussed.

\section{Introduction}

A great number of thermistor linearizing circuits have been proposed. Some of them are based on resistors, while others use active components such as voltage to frequency converters, multipliers, etc.

Hoge (1979) has shown that all circuits based on resistors (serial, parallel, bridge, etc) have the same capacity for linearizing the thermistor characteristic. On the other hand, proposers of active circuits generally present their errors associated with an experimental case. The comparison between different circuits is therefore difficult. Many papers lay claim to very accurate theoretical results. However, the experiments show large errors. All the authors have used the simplest, exponential thermistor model in their theoretical studies. This model gives errors greater than expected for their circuits. For this reason we will analyse the behaviour of better thermistor models.

In section 3 we will calculate the lowest errors produced by resistor linearizing circuits. These values are going to be used as a basis for comparison with more complex circuits.

\section{Models of thermistors}

Equation (1) represents the well known exponential model

$$
R_{\mathrm{t}}=A_{1} \exp \left(B_{1} / T\right)
$$

where $R_{\mathrm{t}}$ is the thermistor resistance and $T$ is the absolute temperature. $A_{1}$ is a parameter that depends on the material and geometry of the thermistor, while parameter $B_{1}$ depends on the material only. The $A_{1}$ and $B_{1}$ values should be selected to obtain the best fit between the model and actual behaviour. In a temperature range of $-10{ }^{\circ} \mathrm{C}$ to $100{ }^{\circ} \mathrm{C}$ the error is about $1 \mathrm{~K}$ (Slomovitz 1987). Figure 1 (curve a) shows a typical error curve. The values are greater than those expected in active circuits. For this reason, it is not possible to calculate circuit errors in a theoretical analysis using the exponential model.

Many models with lower errors than the exponential have been proposed. All of them have three parameters instead of two. This allows better adjustment. Equation (2) shows one of the best models, proposed by Steinhart and Hart (1968, to be referred to as $\mathrm{SH})$.

$$
R_{1}=\exp \left\{\frac{1}{3}\left[\left(-F_{1}+F_{2}\right)^{1 / 3}-\left(F_{1}+F_{2}\right)^{1 / 3}\right]\right\}
$$

where

$$
\begin{gathered}
F_{1}=\frac{27}{2}(\alpha-1 / T) / \gamma \\
F_{2}=\frac{3}{2} \sqrt{3}\left\{27[(\alpha-1 / T) / \gamma]^{2}+4(\beta / \gamma)^{3}\right\}^{1 / 2}
\end{gathered}
$$

$\alpha, \beta$ and $\gamma$ being the three parameters of this model. Unfortunately, from a mathematical point of view it is

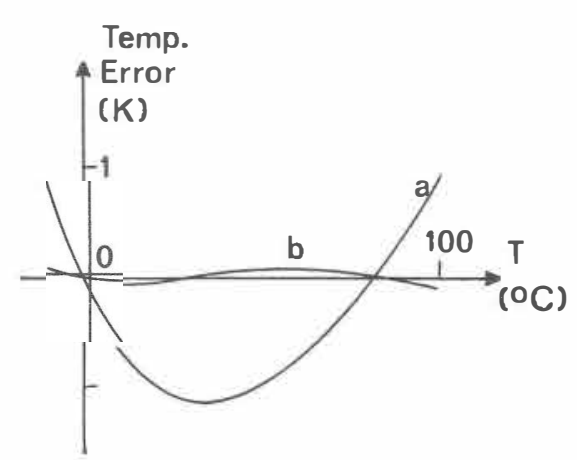

Figure 1. Temperature error in thermistor models: a, exponential model; b, BGS model. 
very cumbersome. Bosson et al (1950, to be referred to as BGS) have proposed another model with similar errors to the $\mathrm{SH}$ model for the temperature range $-10^{\circ} \mathrm{C}$ to $100{ }^{\circ} \mathrm{C}$. When a large range is used $\left(-60{ }^{\circ} \mathrm{C}\right.$ to $\left.150{ }^{\circ} \mathrm{C}\right)$ the error of the BGS model is three times greater than the SH one. However, we prefer to use the BGS model, because the mathematical expression (equation(3)) is simpler than SH. This is an important advantage in theoretical studies.

$$
R_{\mathrm{t}}=A_{2} \exp \left[B_{2} /(T+\theta)\right] .
$$

The $A_{2}, B_{2}$ and $\theta$ parameters must be selected so as to fit the experimental $R_{\mathrm{t}}(T)$ curve as well as possible. A comparison of the error produced by these models in 13 different thermistors has been shown by Slomovitz (1987). The BGS model produces errors 10 to 20 times smaller than the exponential model. A typical error curve is shown in figure 1 (curve b).

The complexity of a theoretical study is increased when a three-parameter model is used. Nevertheless, the BGS parameters are not totally independent. A strong correlation between $B_{2}$ and $\theta$ was observed when these parameters were calculated for the 13 thermistors previously mentioned. Figure 2 shows that this relationship can be approximated by a straight line. The best straight line is

$$
\theta=0.0179 B_{2}-42.7
$$

where $\theta$ and $B_{2}$ are expressed in $\mathrm{K}$. The standard deviation is $12 \%$. This reduces the number of independent variables to two. Thus, studies using this model will not be more complicated than those using the exponential model.

The parameter $B_{1}$ of the exponential model is related to the parameter $B_{2}$ of the BGS model. Figure 3 shows that when $B_{1}$ increases, $B_{2}$ also increases linearly. The points in this figure were calculated using data from the 13 previously mentioned thermistors. The best straight line for these points is given by using

$$
B_{2}=2.332 B_{1}-3268 \text {. }
$$

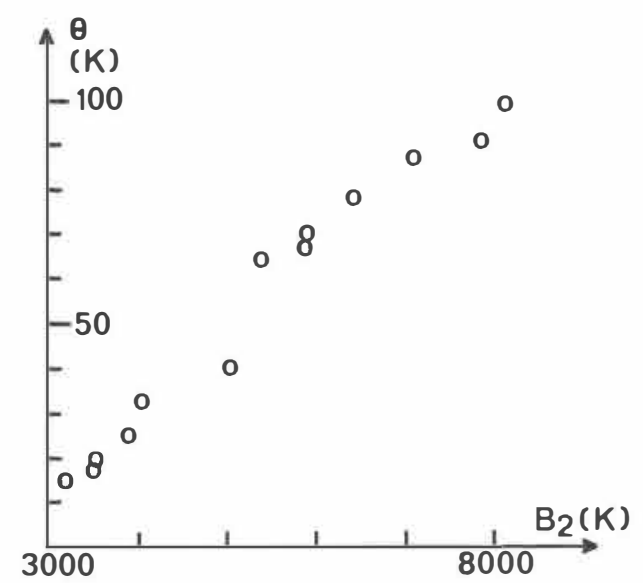

Figure 2. Relationship between the BGs parameters $\theta$ and $B_{2}$.

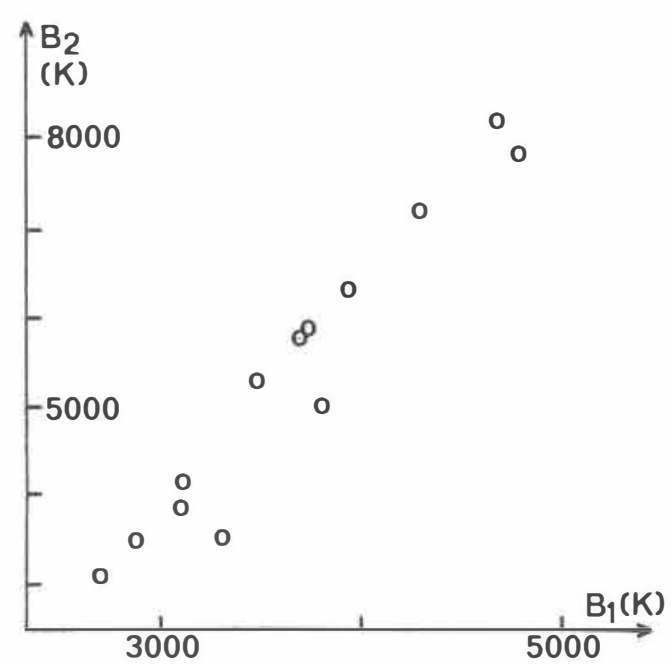

Figure 3. Relationship between the parameters $B_{2}$ (BGS model) and $B_{1}$ (exponential model).

In this way, it is possible to estimate the values of $B_{2}$ and $\theta$ from the value of $B_{1}$. using equations (4) and (5). We will make use of this in section 4.

\section{Single resistor linearizing circuit}

Figure 4 shows one of the simplest linearizing circuits. $U$ is a constant voltage, $V_{\mathrm{o}}$ the output voltage, $R_{\mathrm{t}}$ is the thermistor and $R$ is a resistor.

$$
V_{\mathrm{o}}=U /\left\{\left(A_{2} / R\right) \exp \left[B_{2} /(T+\theta)\right]+1\right\} \text {. }
$$

Figure 5 shows the relationship between $V_{\mathrm{o}}$ and $T$. We define the relative-temperature error $E_{\mathrm{r}}$ as the percentage temperature difference between the curve and the straight line $\left(100 \times(\right.$ segment $\left.\mathrm{GH}) / T_{\mathrm{g}}\right) . T_{\mathrm{g}}$ is given in kelvin. It is necessary, in the temperature range considered, to choose the value of $R$, and to select the straight line that fits the curve best, to reduce errors to a minimum.

Boël et al (1965) have proposed choosing the tangent to the curve at the inflection point (corresponding to $T_{0}$ ), as the straight line. This should produce errors similar to those shown in figure 6 , curve a. The value $T_{0}$ is imposed in the middle of the temperature range by Boël

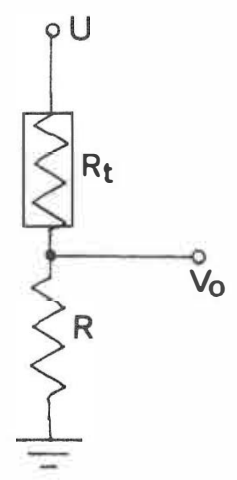

Figure 4. Resistive linearizing circuit of thermistors: $U$, constant voltage; $V_{0}$, output voltage. 


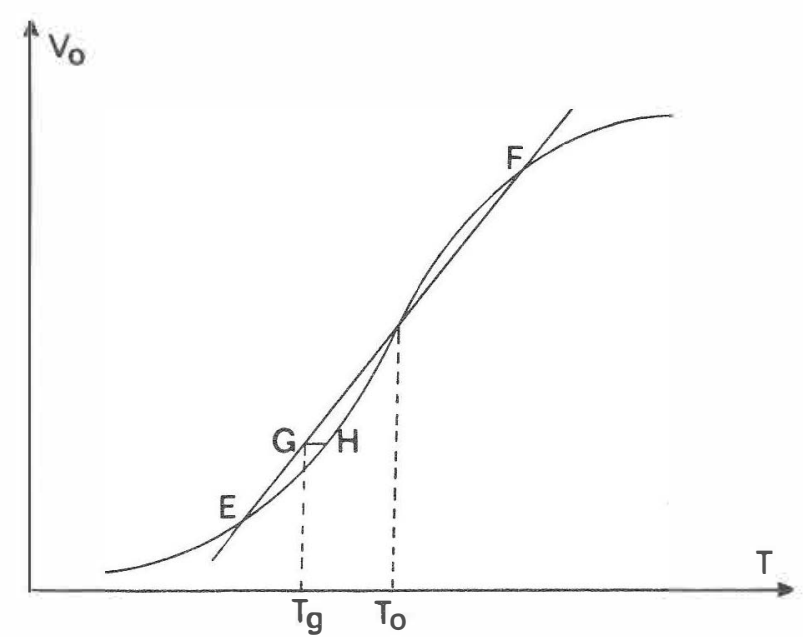

Figure 5. Typical curve of output voltage $V_{0}$ versus the thermistor temperature. $T_{0}$ is the temperature corresponding to the inflection point.

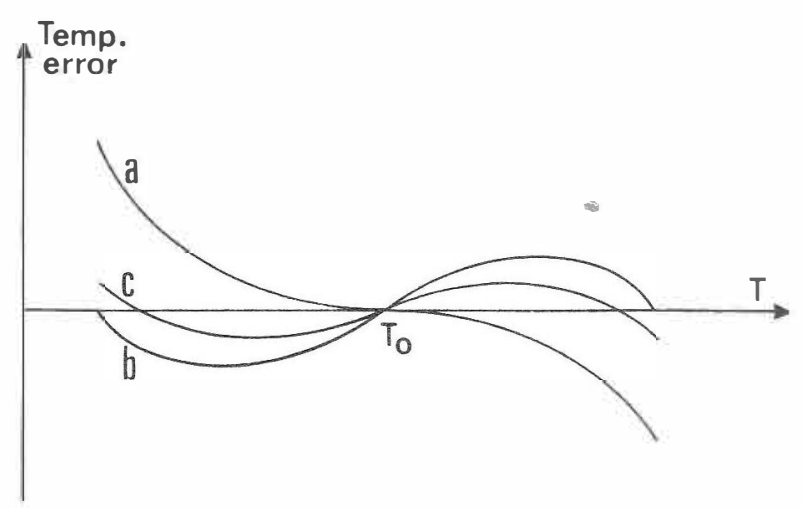

Figure 6. Temperature errors according to different designing methods: a, straight line tangent in the inflection point; $b$, straight line passing through the extremes points; C, straight line passing through $T_{0}$ with a slope between cases $a$ and $b$.

et al. This leads to a condition for the value of $R$. Using the BGS model, this condition is

$$
\begin{aligned}
R_{\mathrm{f}}= & A_{2} \exp \left[B_{2} /\left(T_{0}+\theta\right)\right] \\
& \times\left[B_{2}-2\left(T_{0}+\theta\right)\right] /\left[B_{2}+2\left(T_{0}+\theta\right)\right]
\end{aligned}
$$

Where $R_{\mathrm{f}}$ is the value of $R$ calculated by this method.

Beakley (1951) proposed using the straight line that passes through the two extreme points of the curve. The shape of the curve should be of the type shown in figure 6 , curve b. He proposed choosing the $R$ value according to (7).

Khan (1985) mentioned that smaller errors could be obtained using a straight line that passes through the inflection point, with a slope slightly smaller than the tangent. The shape of the error curve should be similar to curve $\mathrm{c}$ in figure 6. However, he did not show how to choose this slope.

In all these cases, it was a condition that the inflection point of the curve was in the centre of the temperature range, and that the straight line passed through it. However, there is no theoretical reason to support this. We will show later that other criteria lead to smaller errors.
The analytical determination of the $R$ value and the best straight line for an optimal linearization is very cumbersome. These parameters depend on $A_{2}, B_{2}, \theta$ and the temperature range. We use a numerical method to solve this problem. The maximum value of $E_{\mathrm{r}}$ was used as the quality parameter. However, in the other papers the absolute temperature difference $E_{\mathrm{t}}$ (segment $\mathrm{GH}$ in figure 5) is used as the temperature error. For comparison with these other papers, our results are expressed as $E_{t}$, in kelvin.

It was supposed that equation (3) represented exactly the thermistor behaviour. The temperature difference $h$ ( $\left.h=T_{\text {maximum }}-T_{\text {minimum }}\right)$ of the considered range, was varied between $50 \mathrm{~K}$ and $200 \mathrm{~K}$. The thermistor parameters were varied in the following ranges: $B_{2}$ between $3000 \mathrm{~K}$ and $8000 \mathrm{~K}$, and $\theta \pm 20 \%$ around the value given by equation (4). $R$ is proportional to the parameter $A_{2}$, so it is not necessary to take into account the variation of $A_{2}$. A change in the value of $A_{2}$ only produces a proportional change in the value of $R$.

The circuit analysed has three variables. They are $R$, and the two parameters that define the straight line. For these two parameters, we used two of the intersection points between the curve and the straight line (E and $F$ in figure 5). The calculating program varies these three variables, and selects the best values. This method usually uses a lot of computer time, but in this case we know approximately where the solution is. The two intersection points are near the extremes of the temperature interval and the value of $R$ has the same order as the value given by equation (7).

The error $E_{t}$ depends on the temperature difference $h$, but it does not depend on where the range is. Figure 7 shows that different ranges lead to similar errors if $h$ is constant. In this figure, the abscissa represents the central temperature $T_{\mathrm{c}}\left(T_{\mathrm{c}}=\left(T_{\max }+T_{\min }\right) / 2\right)$, and the ordinate the calculated error $E_{t}$. The circles correspond to a thermistor for which $B_{2}=3000 \mathrm{~K}$, the crosses to $B_{2}=5000 \mathrm{~K}$ and the triangles to $B_{2}=8000 \mathrm{~K}$. In all these cases, the value of $\theta$ has been calculated according to equation (4).

The variation of $\theta$ in a range of $\pm 20 \%$ from the central value given by (4), does not alter the errors in practice. Figure 8 shows the variation of the error $E_{\mathrm{t}}$ against the ratio $\theta / \theta_{0}, \theta_{0}$ being the value given by equation (4). Thermistors with three different values of

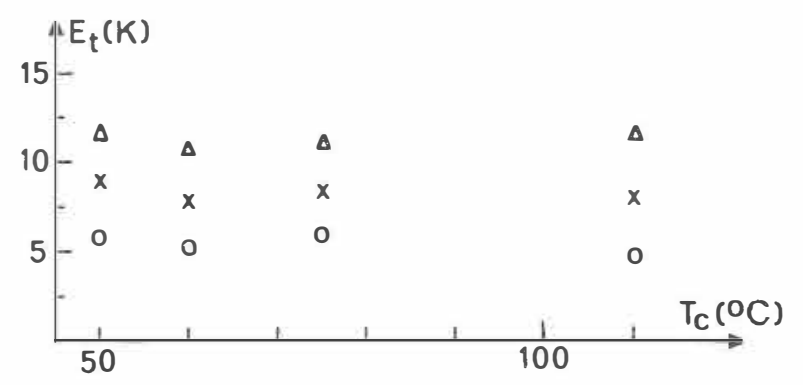

Figure 7. Influence of the central temperature $T_{c}$ on the error $E_{1}$. The temperature difference $h$ is $150 \mathrm{~K}$ for all the points. Circles, $B_{2}=3000 \mathrm{~K}$; crosses, $B_{2}=5000 \mathrm{~K}$; triangles, $B_{2}=8000 \mathrm{~K}$. The value of 0 is given by equation (4). 


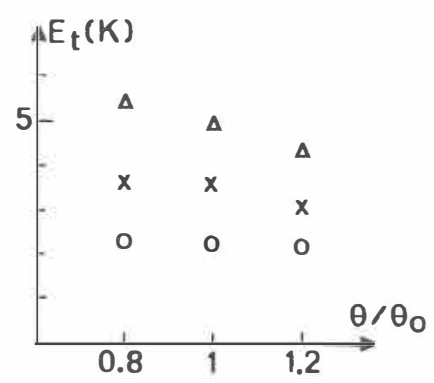

Figure 8. Influence of $\theta / \theta_{0}$ on the error $E_{\mathrm{t}}, \theta_{0}$ being the calculated value according to equation (4).

$B_{2}$ were used (circles, $B_{2}=3000 \mathrm{~K}$; crosses, $B_{2}=5000 \mathrm{~K}$; triangles, $\left.B_{2}=8000 \mathrm{~K}\right)$. $E_{\mathrm{t}}$ varies less than $14 \%$ in all these cases. The temperature range was $0{ }^{\circ} \mathrm{C}$ to $100{ }^{\circ} \mathrm{C}$ for all the points.

Accordingly, errors corresponding to different methods are shown only as a function of $h$ and $B_{2}$. All the temperature ranges begin at $0{ }^{\circ} \mathrm{C}$.

A large number of cases were studied using the numerical method. From the results, a simple heuristic designing method is proposed. This new method is very simple to use. We suggest choosing the value of $R$ according to the heuristic equation

$$
R=k R_{\mathrm{f}}
$$

where $k=0.00313 h+0.913$. $k$ varies between 1.07 for $h=$ $50 \mathrm{~K}$ and 1.54 for $h=200 \mathrm{~K}$. As the straight line, we propose choosing one that produces null errors at temperatures corresponding to $6.9 \%$ and $89.5 \%$ of $h$. As an example, consider a $-10{ }^{\circ} \mathrm{C}$ to $80{ }^{\circ} \mathrm{C}$ range. The value of $h$ is $90 \mathrm{~K}$. There should be null errors at $-3.8^{\circ} \mathrm{C}$ $(-10+0.069 \times 90)$ and $70.6{ }^{\circ} \mathrm{C}(-10+0.895 \times 90)$.

A comparison between the three methods mentioned is presented in table 1 . The proposed method (column 6) has errors which are three times smaller than the Boël method shown in column 4, and 1.6 times lower than the Beakley method shown in column 5. On the other hand, the average error of the proposed method is only 1.09 times greater than the errors generated by the numerical method (column 7).

\section{Error comparison with proposed linearizing circuits}

All authors, at present, have used the exponential thermistor model in their analyses. To compare their results against the proposed circuit, it is necessary to estimate $B_{2}$ and $\theta$ from the value $B_{1}$. For this purpose, we will use equations (4) and (5).

Sundqvist (1983) proposed a voltage to frequency converter. According to his theoretical analysis, based on the exponential model, the error should be zero. Nevertheless, in his experimental results, errors as large as $2 \mathrm{~K}$ appear in a $100 \mathrm{~K}$ range. These errors are attributed to model errors. The thermistor used had $B_{1}=3725 \mathrm{~K}$. This is approximately equivalent to $B_{2}=5420 \mathrm{~K}$. From table 1 it is concluded that the error of a resistive linearizing circuit should be around $3.6 \mathrm{~K}$. This error is of a similar order to the active circuit error. This confirms that the exponential model must not be used with active circuits. Otherwise, the errors should be smaller than those produced by resistive linearizing circuits.

Patranabis et al (1988) proposed using an active circuit based on a logarithmic amplifier. They showed errors around $1 \mathrm{~K}$ in the range $30{ }^{\circ} \mathrm{C}$ to $95^{\circ} \mathrm{C}$. (They did not show the thermistor value of $B_{1}$.) Using a resistive circuit the error should be between $1 \mathrm{~K}$ and $2 \mathrm{~K}$, depending on the thermistor (see table 1). This shows that just a single resistor is equivalent to a complex circuit, so the usefulness of the circuit is doubtful.

Sengupta (1988) proposed a temperature to frequency converter. His experimental results show errors of $0.7 \mathrm{~K}$ in a range of $5^{\circ} \mathrm{C}$ to $85^{\circ} \mathrm{C}$. The thermistor used had $B_{1}=3400 \mathrm{~K}$, corresponding to $B_{2}=4600 \mathrm{~K}$. Table 1 shows that a resistive circuit has errors of around $2 \mathrm{~K}$ for the same conditions. For applications where this slightly higher error is acceptable, the resistive circuit offers a much more cost-effective solution than the active circuit.

On the other hand, Cole (1957) used a resistive circuit. During the experimental work, he empirically adjusted the resistor values and the straight line to obtain the smallest error. The range considered was $2{ }^{\circ} \mathrm{C}$ to $48{ }^{\circ} \mathrm{C}$. He imposed null errors at $5{ }^{\circ} \mathrm{C}$ and $45^{\circ} \mathrm{C}$. These values

Table 1.

\begin{tabular}{|c|c|c|c|c|c|c|}
\hline $\begin{array}{l}B_{2} \\
(K)\end{array}$ & $\begin{array}{l}\text { Range } \\
\text { (K) }\end{array}$ & $\begin{array}{l}h \\
(\mathrm{~K})\end{array}$ & $\begin{array}{l}\text { Method } 1 \\
\text { (Boël) } \\
E_{t}(K)\end{array}$ & $\begin{array}{l}\text { Method } 2 \\
\text { (Beakley) } \\
E_{\mathrm{t}}(\mathrm{K})\end{array}$ & $\begin{array}{l}\text { Method } 3 \\
\text { (proposed) } \\
E_{\mathrm{t}}(\mathrm{K})\end{array}$ & $\begin{array}{l}\text { Numerica } \\
\text { method } \\
E_{\mathrm{t}}(\mathrm{K})\end{array}$ \\
\hline 3000 & $273-323$ & 50 & 1.3 & 0.6 & 0.4 & 0.3 \\
\hline 3000 & $273-373$ & 100 & 7.5 & 4.2 & 2.5 & 2.3 \\
\hline 3000 & $273-423$ & 150 & 17.8 & 10.6 & 6.3 & 5.9 \\
\hline 3000 & $273-473$ & 200 & 29.2 & 19.4 & 12.3 & 9.9 \\
\hline 5000 & $273-323$ & 50 & 2.2 & 1.0 & 0.6 & 0.6 \\
\hline 5000 & $273-373$ & 100 & 11.9 & 6.3 & 3.6 & 3.6 \\
\hline 5000 & $273-423$ & 150 & 28.8 & 15.7 & 9.3 & 8.5 \\
\hline 5000 & $273-473$ & 200 & 51.6 & 26.8 & 17.5 & 16.1 \\
\hline 8000 & $273-323$ & 50 & 3.0 & 1.4 & 0.9 & 0.9 \\
\hline 8000 & $273-373$ & 100 & 16.4 & 8.2 & 4.8 & 4.8 \\
\hline 8000 & $273-423$ & 150 & 38.9 & 20.3 & 12.2 & 11.0 \\
\hline 8000 & $273-473$ & 200 & 68.1 & 35.4 & 22.7 & 20.8 \\
\hline
\end{tabular}


are very close to those recommended for the proposed method $\left(5^{\circ} \mathrm{C}\right.$ and $\left.43^{\circ} \mathrm{C}\right)$. The thermistor used had $B_{1}=$ $3463 \mathrm{~K}$, corresponding to $B_{2}=4800 \mathrm{~K}$. The error obtained, $E_{t}$, by Cole was $0.7 \mathrm{~K}$. Table 1 forecasts $0.6 \mathrm{~K}$. This shows that the theoretical errors shown in table 1 , column 6 , can be obtained in real experiments.

\section{Conclusions}

A method for designing resistive linearizing circuits has been proposed. It is based on a three-parameter thermistor model (Bosson et al 1950). Analysis of this model shows that there is a strong correlation between the parameters. Also, simple equations relate these parameters to the exponential model parameters.

An error comparison between the proposed method and active circuits was done. It is surprising that a lot of recent papers show errors of the same order as those produced by single resistor circuits. The principal cause is that all of them are based on the simple exponential approximation. For this reason they cannot be expected to be perfect.

We propose using the results of this paper as a basis for comparison of the quality of active linearizing circuits.

\section{References}

Beakley W R 1951 The design of thermistor thermometers with linear calibration J. Sci. Instrum. 28 176-9

Boël M and Erickson B 1965 Correlation study of a thermistor thermometer Rev. Sci. Instrum. 36 904-8

Bosson G, Gutmann F and Simmons L M 1950 A relationship between resistance and temperature of thermistors J. Appl. Phys. 21 1267-8

Cole K S 1957 Thermistor thermometer bridge: linearity and sensitivity for a range of temperature Rev. Sci. Instrum. $28326-8$

Hoge H J 1979 Comparison of circuits for linearizing the temperature indications of thermistors Rev. Sci. Instrum. $50316-20$

Khan A A 1985 Linearization of thermistor thermometer Int J. Electron. 59 129-39

Patranabis D, Ghosh S and Bakshi C 1988 Linearizing transducer characteristics IEEE Trans. Instrum. Meas. 37 $66-9$

Sengupta R N 1988 A widely linear temperature to frequency converter using a thermistor in a pulse generator IEEE Trans. Instrum. Meas. 37 62-5

Slomovitz D 1987 The temperature/resistance curve of NTC thermistors Test and Measurement World 7 (5) 73-9

Steinhart J S and Hart S R 1968 Calibration curves for thermistors Deep-Sea Res. 15 497-503

Sundqvist B 1983 Simple, wide-range, linear temperature-tofrequency converters using standard thermistors $J$. Phys. E: Sci. Instrum. 16 261-4 